\title{
SOME ASPECTS OF MODERN PHOTOGRAMMETRIC IMAGE PROCESSING OF SOVIET LUNOKHOD PANORAMAS AND THEIR IMPLEMENATION FOR NEW STUDIES OF LUNAR SURFACE
}

\author{
N. A. Kozlova ${ }^{1}$, A. E. Zubarev ${ }^{1}$, I. P. Karachevtseva ${ }^{1}$, I. E. Nadezhdina ${ }^{1}$, A. A. Kokhanov ${ }^{1}$, \\ V. D. Patraty ${ }^{1}$, L. A. Mitrokhina ${ }^{1}$, J. Oberst $^{2}$
}
${ }^{1}$ Moscow State University of Geodesy and Cartography (MIIGAiK), MIIGAiK Extraterrestrial Laboratory (MExLab), Gorokhovskiy per., 4, 105064, Moscow, Russia, e-mail: icar2003@mail.ru
${ }^{2}$ German Aerospace Center (DLR), Germany

Commission IV, Working Group IV/8

KEY WORDS: Moon, Panorama processing, Soviet Lunar Missions, Lunokhods, Surface Modelling, LRO NAC

\begin{abstract}
:
Recently the Lunar Reconnaissance Orbiter (LRO) has obtained images, which cover landing sites from previous missions to the Moon in unprecedented high resolution (up to $0.3 \mathrm{~m} / \mathrm{pixel}$ ). These new data allow us to carry out a precision mapping of the Lunokhod landing sites, combining orbital high resolution images with surface panoramas taken by the rovers during their missions. Unfortunately, a lot of supplementary information about the missions, the rovers and their instrumentation is lost, which includes timing information and exterior orientation parameters. So, we have developed new methods and techniques of Lunokhod panorama processing by means of modern photogrammetric software supported by LRO data
\end{abstract}

\section{INTRODUCTION}

Russia has a long tradition in Moon exploration. Early highlights of lunar research include the first images of the Moon far side and the first soft landings. Also, the historic Lunokhods were the first planetary rovers, extremely successful in their early missions: Lunokhod-2 has completed a traverse of more than $40 \mathrm{~km}$.and has transferred about 300 panoramas of the lunar surface to Earth. These data was used for operational decision-making during the missions, and for science and exploration purposes. Unfortunately, only small portions of the data have been processed and are available for the scientific community in digital form. Recently the Lunar Reconnaissance Orbiter (LRO) has obtained Moon images in unprecedented detail (up to $0.3 \mathrm{~m} /$ pixel) that cover all landing sites from previous American and Soviet missions. Our main goal is to convert lunar panoramas to formats useful for measurements, taking advantage of modern photogrammetry image processing, GIS tools, and stereo images obtained by the LRO Narrow Angle Camera (LRO NAC). Likewise, we are investigating DEMs and orthoimages of the landing sites.

Results of the processing of archive panoramas will be used in EU FP7 PRoViDE project (Planetary Robotics Vision Data Exploitation) which aims to assemble the imaging data gathered from probes and rovers on planetary surfaces into a unique database, bringing them into a spatial context and to provide open access to a complete set of $3 \mathrm{D}$ vision products (http://www.provide-space.eu/).

\section{METHODOLOGY}

\subsection{Available data and software}

MIIGAiK has recently obtained archive panoramas from the State Archive of Russian Federation for research purposes, including those from the missions Luna 9, 13, 17, 20, 21. Three of them (Luna 9, 13 and 20) were stationary lander missions, where only a few panoramas were taken. The other two missions (Luna 17, 21) had rovers - Lunokhod-1 and Lunokhod-2, respectively. Panoramas taken by the rovers over their wide activity area are highly useful for geomorphologic analyses of the different types of lunar surfaces.

Commercial photogrammetric software PHOTOMOD (Racurs ${ }^{\mathrm{TM}}$, http://www.racurs.ru/?ng=en\&page=634) is used for photogrammetric processing. For transformation and projecting of assembled panoramas special software has been developed.

\subsection{Description of Lunokhod's cameras}

Each Lunokhod had two panoramic cameras on each side (one horizontal and one vertical). Horizontal cameras obtained nearhorizontal views, the width of panorama is $30^{\circ}$ (500 samples) and length is a bit more than $180^{\circ}$ (3000 lines). The optical axes of horizontal cameras were diverted from the horizon by $15^{\circ}$ downwards (Fig. 1). Horizontal panoramas are the main source of information for analyzing the structure of the lunar regolith and relief. Also, topographic surveys were made. A stereophotogrammetric survey of the surrounding area was usually accomplished by means of taking panoramas from two positions of the Lunokhod (Vinogradov, 1971). The vertical cameras have a field of view $30^{\circ} \times 360^{\circ}$ and can capture the sky, the Earth and the Sun, Lunokhod wheels, antennas, and a special device used as an indicator of gravity vector orientation (plumb line measurement or Lunokhod tilt-meter).

\footnotetext{
* Corresponding author.
} 


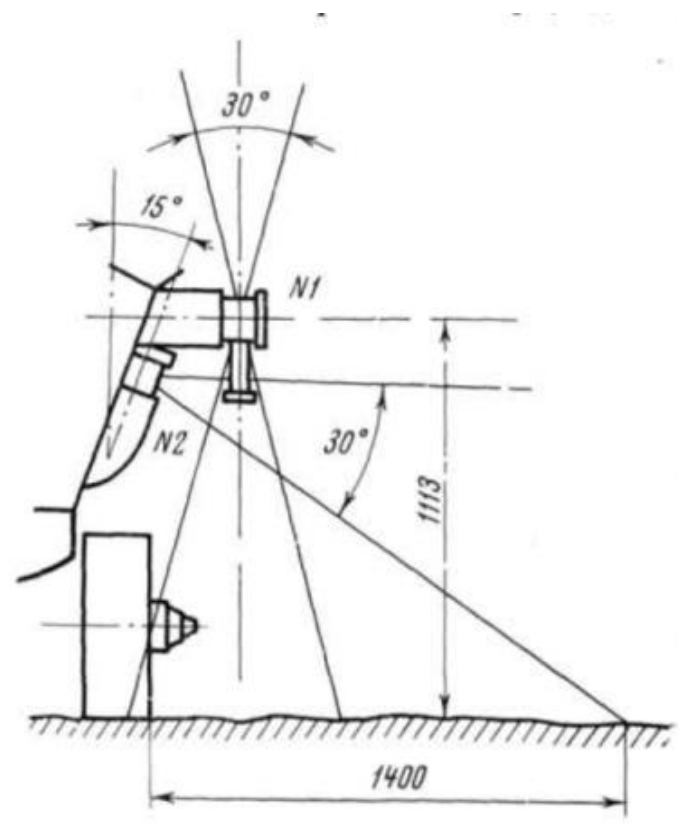

Fig. 1. Scheme of field of view of vertical (N1) and horizontal (N2) panoramic cameras (Vinogradov, 1971)

Panoramic sweeps of the surroundings by these cameras were made with the help of a scanning mirror, which performed oscillatory and rotating motion. So, a panoramic image represents a section of a sphere (spherical projection - see Fig. 2 ). As the geometry is non-standard, panoramas have to be transformed into central projection for further stereo photogrammetric processing.

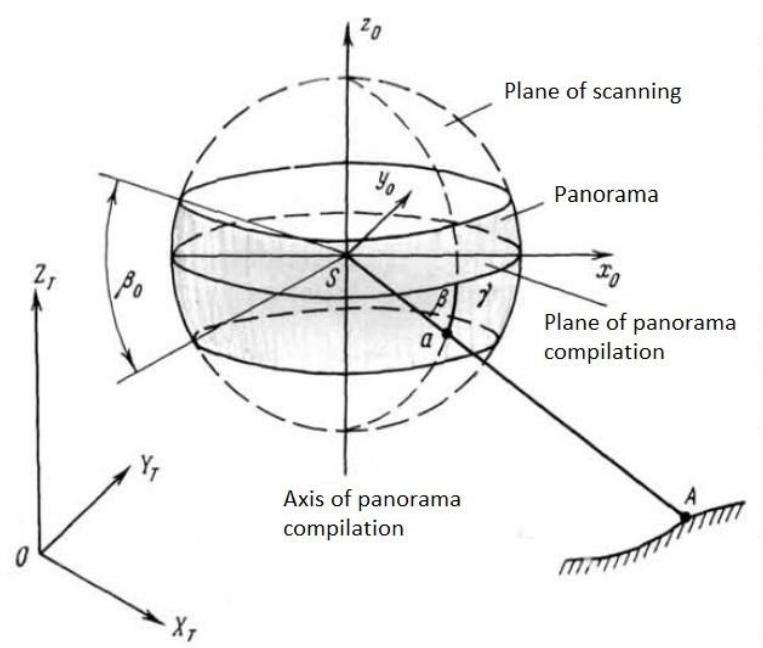

Fig. 2. Scheme of construction of panoramic images

(Vinogradov, 1971)

\subsection{Preparation of panoramas}

The objectives of our work are to fully exploit the historic Lunokhod data based on LRO NAC photogrammetry image processing and use the results for landing site selection for future lunar missions. Unfortunately, many of the relevant operational parameters of the Lunokhod missions are lost. The images are noisy, and image dynamics is low. Timing and positional information, as well as the geometric properties of the cameras are not known and must be determined in the process.

We have developed an algorithm for reconstruction of unknown exterior orientation and processing of panoramas that allowed us to search for stereo pairs and obtain a stereo model from panoramas (where possible).

First of all, we need to assemble panoramas as we obtained them from Russian State Archive in form of scanned fragments. Then we resample them close to their original size (scanned panoramas have a size 5 times larger than original images). Again, assembled panoramas are initially in spherical projection. Besides that there are some distortions caused by non-uniform rotation of the scanning mirror (marked with red arrows in Fig. 3).

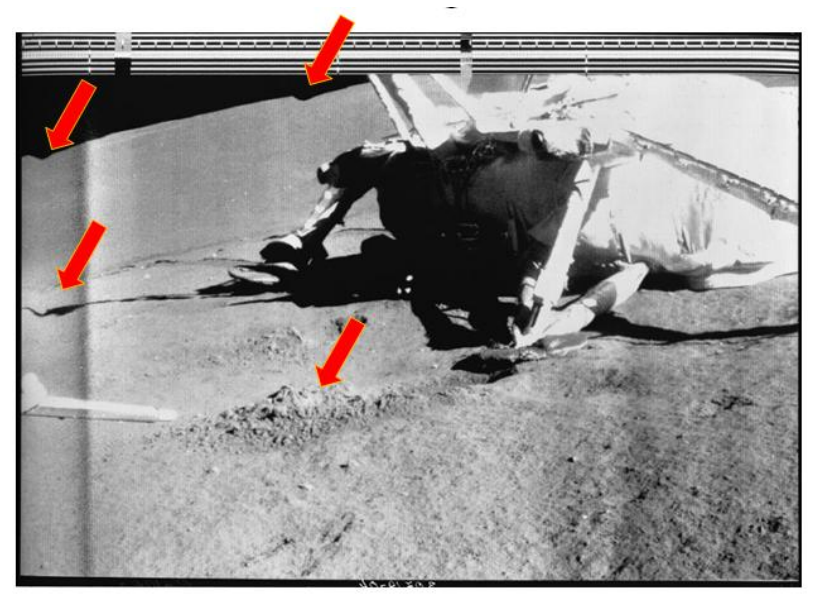

Fig. 3. Image distortions caused by non-uniform rotation of the scanning mirror

\subsection{LRO NAC image processing}

We have created a detailed DEM with a grid size of $1 \mathrm{~m}$ from stereo photogrammetric processing of two LRO NAC stereopairs (M150756018 and M150749234 (left and right images)) (Zubarev et al., 2012; Zubarev et al., 2013). The DEM covers the entire area of the Lunokhod-1 track. We have also selected and orthorectified (using the created DEM) LRO NAC images with the best resolution and illumination conditions for this region. These products (as well as DEM and orthomosaic obtained by DLR) were used to carry out studies of the Lunokhod-1 traverse and morphologic assessments of the working area (Karachevtseva et al., 2013).

We have also created a DEM for Lunokhod-2 working area but its resolution is reduced over that for the Lunokhod- 1 area. This is so because the Lunokhod-2 route is more than 4 times longer and is elongated from West to East. So, to cover the area, several NAC stereo pairs are needed. Also conditions of these stereo images are not as good as for the first Lunokhod: resolution, stereo angle and relative accuracy of the spacecraft coordinates are limited. The work on improvement of the Lunokhod-2 DEM is in progress. 


\section{DETERMINATION OF EXTERIOR ORIENTATION OF PANORAMIC IMAGES}

\subsection{The problem of unknown parameters}

Further processing of panoramas is complicated by lack of some camera parameters and their calibration (principal point, distortion, other parameters, e.g. focal length and image size, not defined precisely), exterior orientation parameters (coordinates of observation points; Lunokhod orientation azimuth and tilts), parameters of digitizing. So for panoramic image processing we have to use nominal interior orientation parameters of the cameras published in (Vinogradov, 1971) or some best fit parameters determined iteratively. In order to determine exterior orientation of the panoramas we have developed two different ways.

\subsection{The first way of determination of exterior orientation}

We have developed a special program, which allows us to convert assembled panoramas (Fig. 4a) to spherical projection to the horizon, in other words, to correct for the tilt of the camera while scanning (Fig. 4b). Determination of the tilts is an iterative process.

\section{a)}

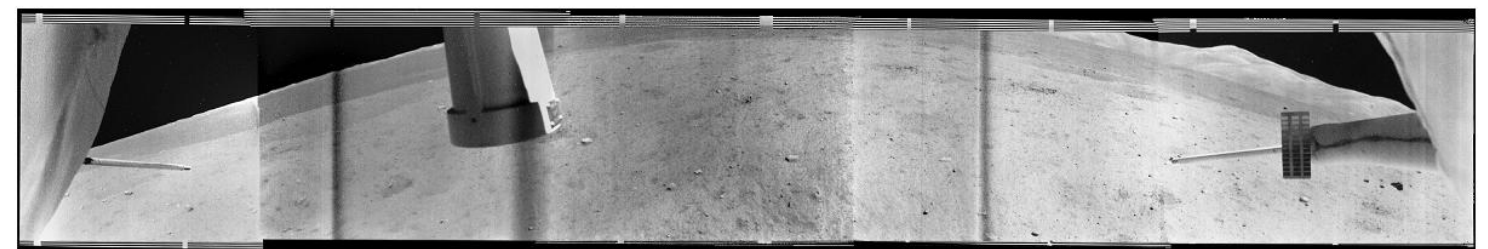

b)

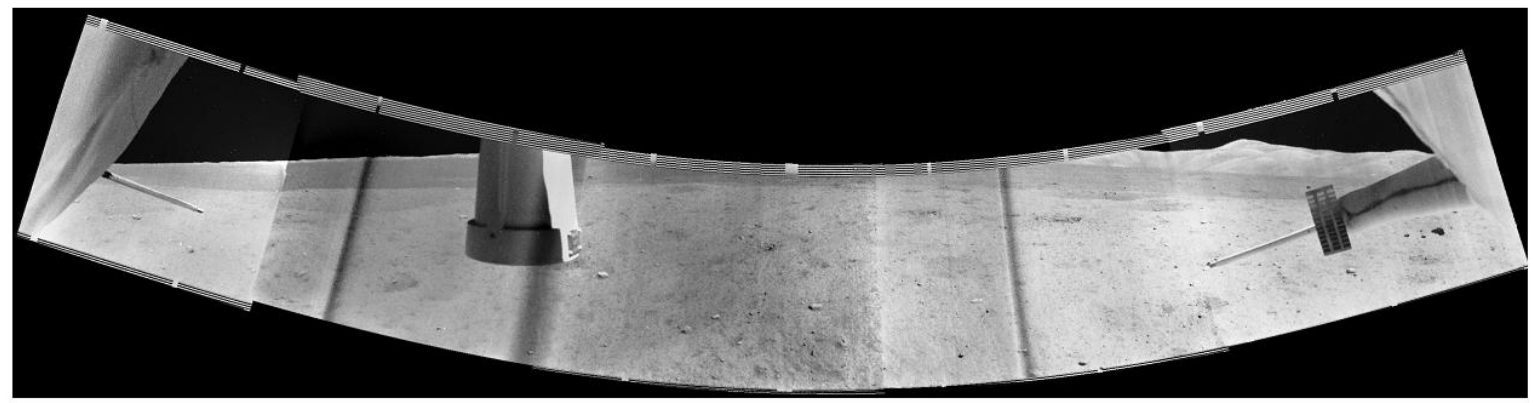

c)

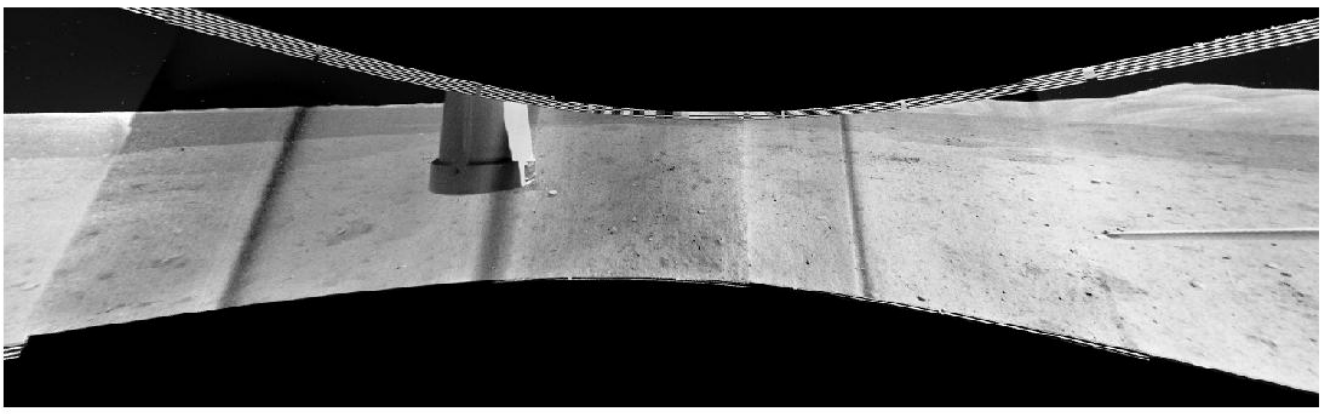

Fig. 4. Lunokhod-2 panorama a) assembled from archive fragments (spherical projection); b) assembled and brought to the horizon (spherical projection) with the following tilts of the panorama: cross tilt $-17.0^{\circ}$ (downwards the surface), longitudinal tilt $-2.0^{\circ}$; c) assembled, brought to the horizon, and transformed into central projection (same tilts).

Another module of the program can transform the panorama from spherical into central projection using the determined tilts (Fig. 4c). Stereo pairs of panoramic images in central projection may be used for stereo photogrammetric processing and creation of DEMs of the lunar surface. Then, in another module of this program it is possible to create an orthoimage from the panorama (Fig. 5a). The geometric quality of orthoimage also works as a control of determined tilts: visible track and craters should be geometrically correct. However, sometimes, local relief affects the resulting image significantly. The last step in this way is to pinpoint the observation sites on orthorectified LRO NAC images - to fit orthorectified panoramas to the LRO NAC orthoimage manually (Fig. 5b).

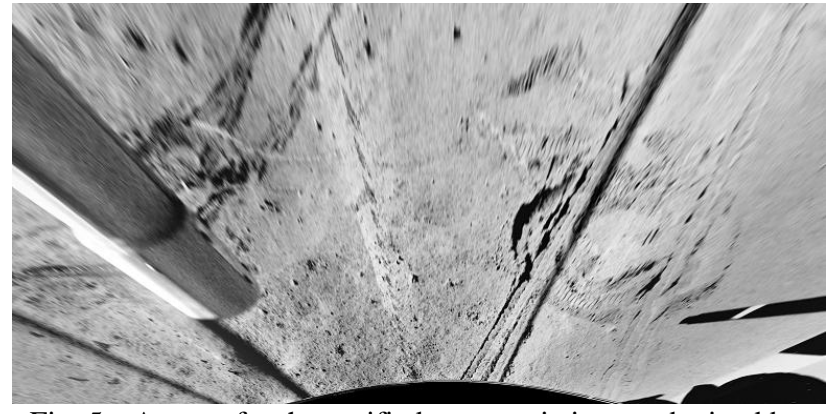

Fig. 5a. A part of orthorectified panoramic image obtained by Lunokhod-2. 


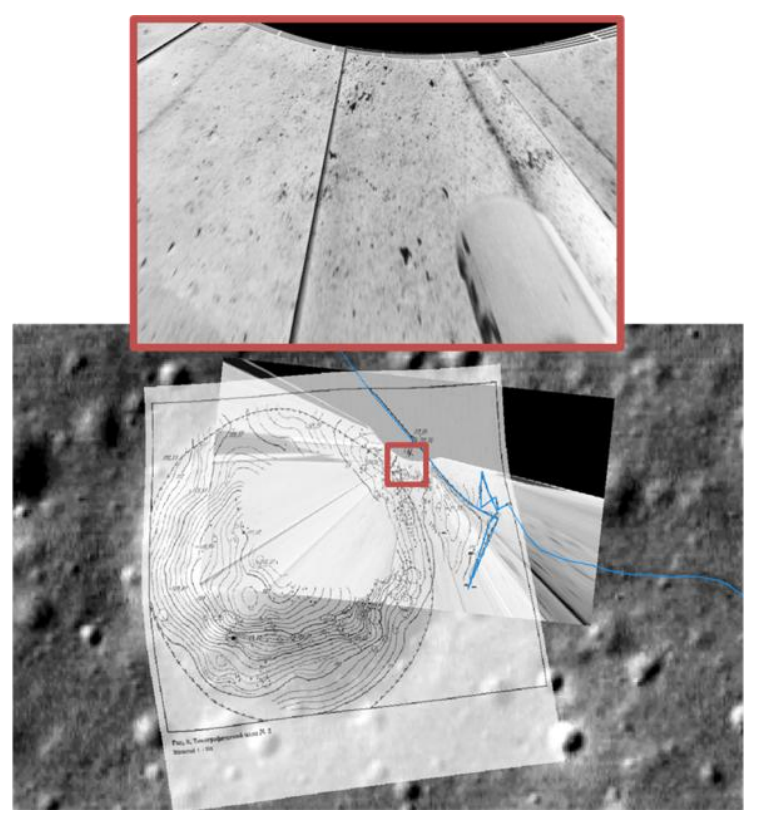

Fig. 5b. Orthorectified panorama (Lunokhod-1) fitted to the LRO NAC and to the topographic plan published in (Barsukov, 1978)

Any information on the Lunokhod position or the exact observation time could be very helpful. But for most of the panoramic images we know only the lunar day of image acquisition. Note that during lunar daylight (14 Earth days) Lunokhod-2 could cover a distance of up to $10 \mathrm{~km}$. Moreover, practically everything what is seen on panoramas is too small to be identified on LRO images (which resolution is 0.3-0.5 $\mathrm{m} /$ pixel while we can see details up to several $\mathrm{cm}$ in the foreground of panoramas).So we create detailed LRO NAC DEMs and orthoimages to reconstruct Lunokhod traverses precisely and evaluate possible observation points and directions.

\subsection{The second way of determination of exterior orientation}

The second way to determine exterior orientation is to model panoramas for several possible observation points (based on the LRO NAC DEM and orthomosaic and using possible tilts) and then compare the artificially modelled panorama (Fig. 6) with the assembled one (Fig. 7). If they seem to be identical (after several iterations) we tie the exterior orientation parameters to the assembled panorama. We also try different methods of visualization of DEMs available in different software packages (e.g. ArcScene, PHOTOMOD), which facilitates preliminary determination of observational point and azimuth (Fig. 8). Especially it is helpful for panoramas which capture hills on the background (Lunokhod-2 mission) but have few prominent objects in the foreground.

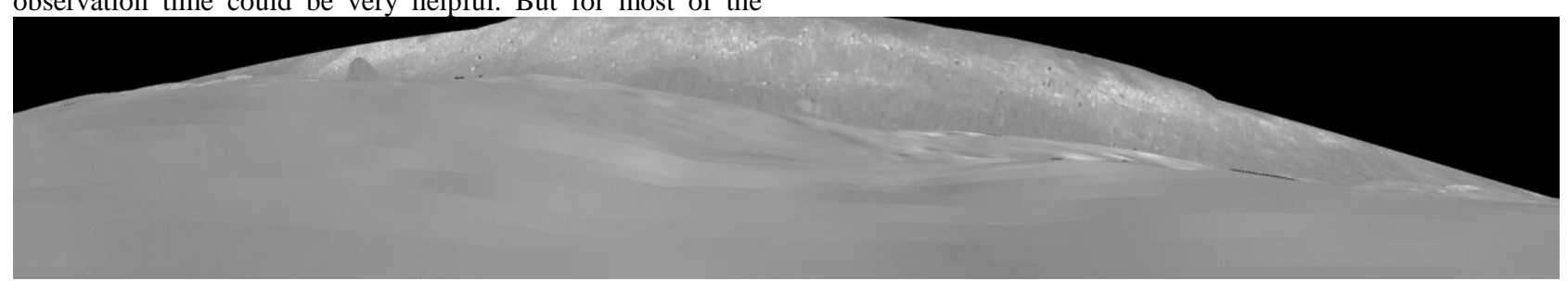

Fig. 6. Panoramic image modeled at MExLab using LRO NAC orthoimage and DEM

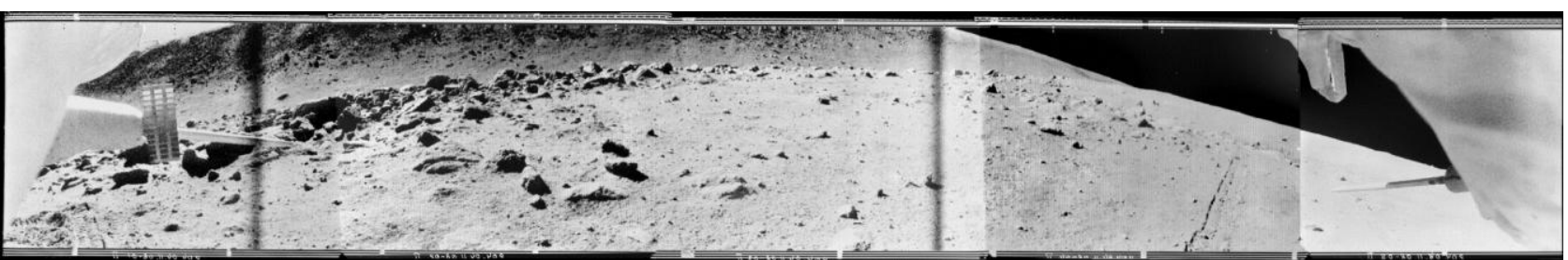

Fig. 7. Assembled archive panorama (Lunokhod-2) for approximately same region as modeled panorama in Fig. 6.

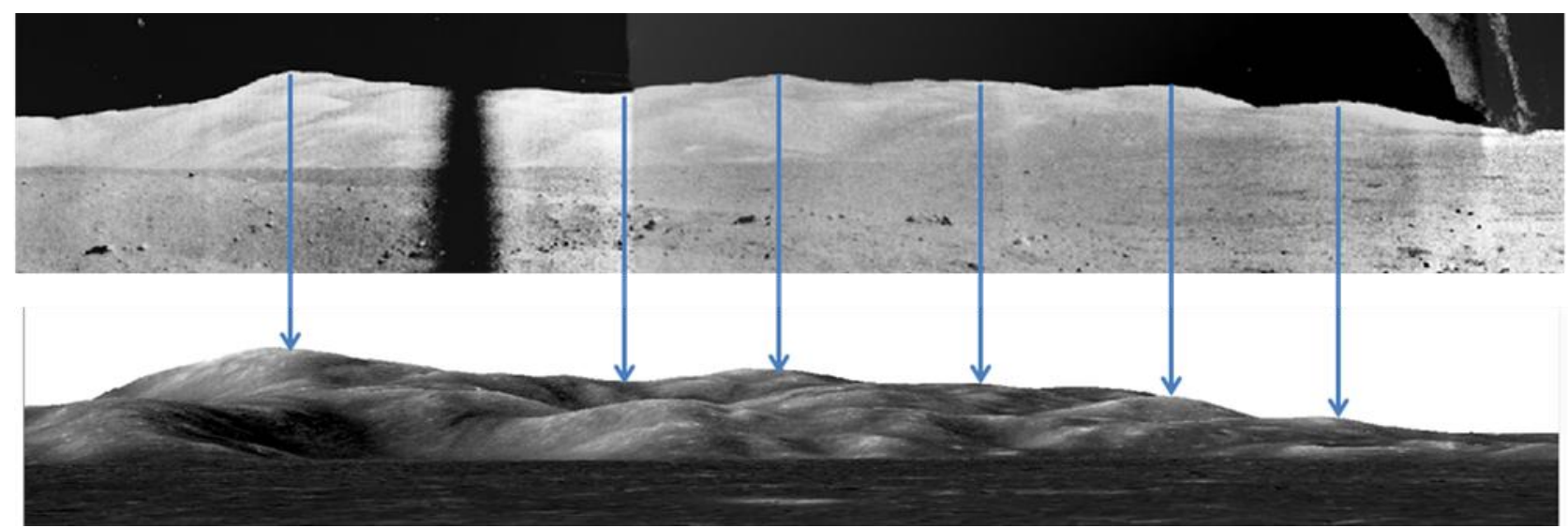

Fig. 8. The image at the top is part of an archive panorama, assembled, and corrected for horizon geometric distortion (Lunokhod-2). Below is ArcScene 3D visualization of Kaguya DEM and orhtomosaic (Haruyama et al., 2012) for the same region. Blue arrows indicate the identical relief features. 


\section{PROPOSED RESULTS AND PRODUCTS}

As a result of our study we expect to obtain the following products (Fig. 9):

1. Archive panorama image fragments and their archive description (Level 0).

2. Archive panorama image fragments with their corrected and extended description (Level 1).

3. Panoramas assembled by means of PHOTOMOD with halfautomatic local brightness balance and produced close to the original size and resolution (Level 2).

4. Panoramas corrected for horizon geometric distortion (in original spherical projection and central projection, Level 3).

5. Orthotransformation of panoramas on sphere or orthorectification using LRO NAC DEM (Level 4).

6. Stereo models obtained from high quality stereo panoramic images, if any will be found (Level 5).

7. Panoramas orthorectified using relief from the stereo model (Level 6).

Every product will be presented in a digital form suitable for its further analyses in most of the modern software packages (e.g. ArcGIS, etc.) and will be uploaded into a database. For this purpose we have also worked out a special scheme of catalogue with metadata for each level of products. The catalogue will include all obtained and recovered information about panoramas, cameras and missions. Metadata will be created for each image based on the catalogue information using the PDS4 standard.

\section{CONCLUSIONS}

We have developed the capability to study historic lunar panoramic images in combination with high-resolution LRO data. We derived terrain models and orthoimages of the sites for data fusion with the panoramic images obtained in-situ from the lunar surface. The fully reconstructed panoramas obtained by new photogrammetric processing techniques (Mitrokhina et al., 2013) provide morphology information, which can be used for quantitative surface analyses of the Lunokhod areas, e.g., for detailed mapping, statistics of rocks, and morphometric descriptions of small craters. Also, we plan to determine physical parameters of lunar regolith based on the depth of Lunokhod tracks. Where stereo images are available, we will produce topographic models by photogrammetric processing. The results of our work, including the rebuilt archival panoramas, will be placed into a MIIGAiK planetary database and will be available via a Geo-portal (http://cartsrv.mexlab.ru/geoportal/). After all panoramas are processed and geolocated, we plan to continue our study (Karachevtseva et al., 2013) of the Lunokhod area in more detail.

Russia is now preparing new Lunar missions. The Russian space agency Roskosmos approved a new space program, which includes Lunar landing missions: the small lander Luna-25 ("Luna-Glob") for testing new technologies (launch planned for
2016), orbital missions for global mapping Luna-26 ("LunaGlob-Orbiter", 2018) and the large lander Luna-27 ("LunaResource") for scientific investigations (2019). Both landers will be landing in the South polar region of the Moon, and it is necessary to carry out a full and detailed study of the characteristics of the planned landing sites. Despite the large amount of modern LRO NAC images for the south sub-polar area, there is a limited number of useful images for creation of DTMs. For landing site selection, we therefore propose to use panoramas and morphometric analyses from the Lunokhod sites and extrapolate our assessments to the lunar polar areas (Basilevsky et al., 2014).

\section{References}

Basilevsky A.T., Kreslavsky M.A., Karachevtseva I.P., Gusakova E.N. Morphometry of small impact craters in the Lunokhod-1 and Lunokhod-2 study areas. 2014. Elsevier, Planetary and Space Science/

Barsukov V. L. (ed.) Mobile laboratory on the Moon Lunokhod-1, Vol. 2. Nauka, 1978 (in Russian).

Haruyama J., Hara S., Hioki K., et. al. LUNAR Global Digital Terrain Model Dataset Produced From Selene (Kaguya)Terrain Camera Stereo Observations // Abstract 43 LPSC2012-1200.

Karachevtseva I., Oberst J., Scholten F., Konopikhin A., Shingareva K., Cherepanova E., Gusakova E., Haase I., Peters O., Plescia J., Robinson M. Cartography of the Lunokhod-1 Landing Site and Traverse from LRO Image and Stereo Topographic Data. 2013 Elsevier, Planetary and Space Science, Vol.85, p.175-187.

Mitrokhina L., A. Zubarev, I.Nadezhdina, I. Karachevtseva. Recovery of exterior orientation of Lunokhod panoramic images using LRO terrain models. International Society for Photogrammetry and Remote Sensing (ISPRS) Meeting of the Working Group IV/8 "Advances in Planetary Mapping and Spatial Databases", MIGAiK, Moscow, Russia, October 9-12, 2013 (oral)

Vinogradov A.P. (ed.) Mobile laboratory on the Moon Lunokhod-1, Vol. 1. Nauka, 1971 (in Russian)

Zubarev A., I. Nadezhdina, N. Kozlova, I. Karachevtseva, J. Oberst, M. Robinson "High-resolution terrain models from LRO stereo images for Luna-Glob landing site selection" The Third Moscow Solar System Symposium (3M-S3), Space Research Institute, Moscow, Russia, 2012, abs. 3MS3-PS-13. http://ms2012.cosmos.ru/sites/ms2012.cosmos.ru/files/3m-s3abstracts.pdf

Zubarev A., I. Nadezhdina, N. Kozlova, I. Karachevtseva, E. Gusakova, J. Oberst "Lunokhod-1 Panoramic Images and Stereo Topography" European Planetary Science Congress 2012, Madrid, Spain, abstract EPSC \#477. 


\section{Acknowledgements}

The research leading to these results has partly received funding from the European Community's Seventh Framework Programme (FP7/2007-2013) under grant agreement № 312377 PRoViDE. Work on Geoportal was supported with a grant of the Ministry of Education and Science of the Russian Federation for "Development of a Planetary Data Geoportal to provide access to results of research on planets and satellites of Solar system" (14.B37.21.1303).

We also would like to acknowledge Russian State Archive of Scientific and Technical Documentation which provided image fragments of archive panoramas for the research.

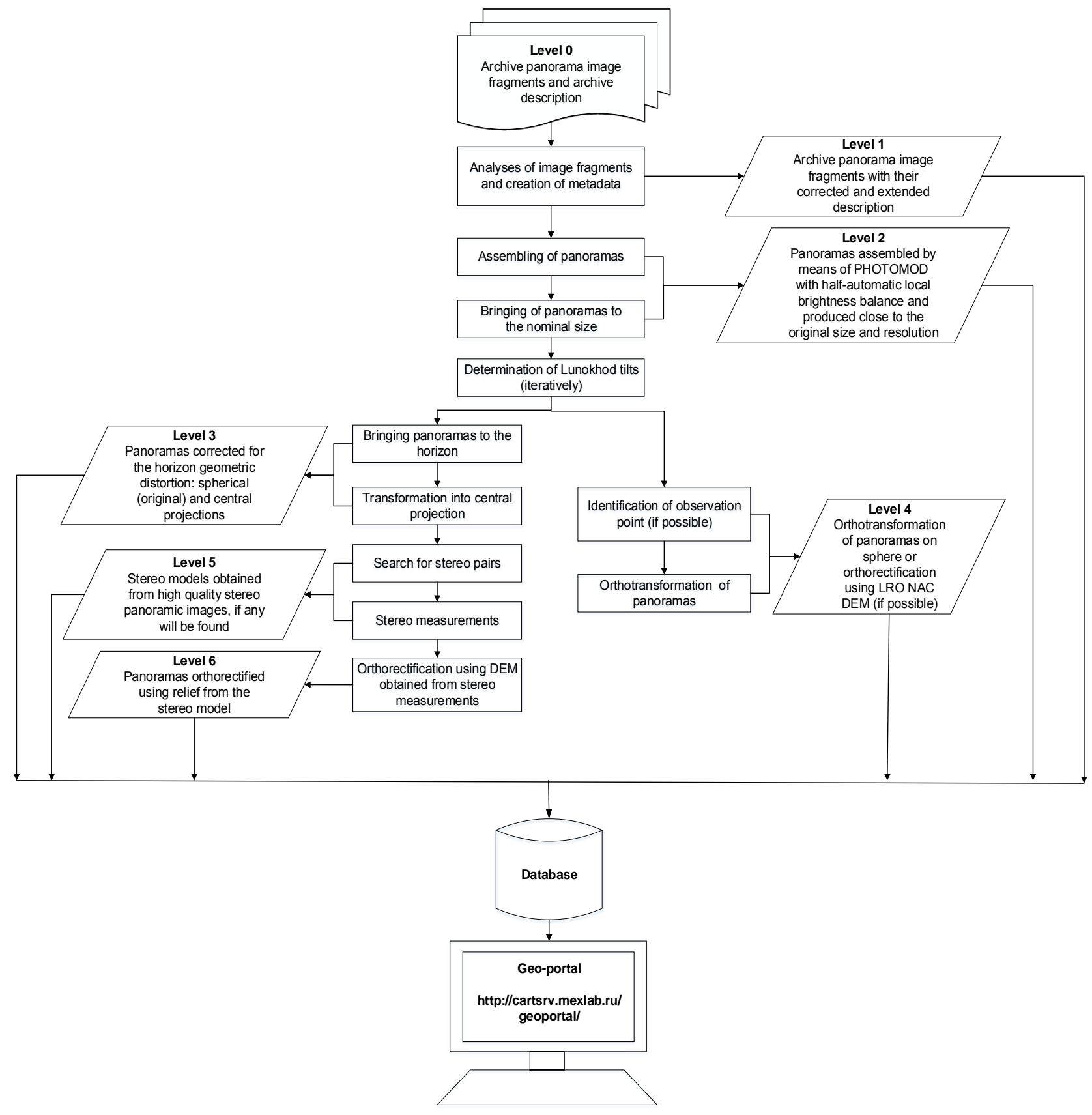

Fig. 9. Scheme of data processing 